\title{
The distributed p-median problem in computer networks
}

Conference or Workshop Item

Accepted Version

AlDabbagh, A., Di Fatta, G. and Liotta, A. (2019) The distributed p-median problem in computer networks. In: ICCSA 2019 Conference, 1-4 Jul 2019, Saint Petersburg, Russia, pp. 541-556. doi: https://doi.org/10.1007/978-3-030-24311-1_39 Available at https://centaur.reading.ac.uk/86457/

It is advisable to refer to the publisher's version if you intend to cite from the work. See Guidance on citing.

Published version at: http://dx.doi.org/10.1007/978-3-030-24311-1_39

To link to this article DOI: http://dx.doi.org/10.1007/978-3-030-24311-1_39

All outputs in CentAUR are protected by Intellectual Property Rights law, including copyright law. Copyright and IPR is retained by the creators or other copyright holders. Terms and conditions for use of this material are defined in the End User Agreement.

\section{www.reading.ac.uk/centaur}

\section{CentAUR}

Central Archive at the University of Reading

Reading's research outputs online 


\title{
The Distributed p-Median Problem in Computer Networks
}

\author{
Anas AlDabbagh \\ Department of Computer Science, University of Mosul, Iraq \\ Giuseppe Di Fatta \\ Department of Computer Science, University of Reading, UK
}

Antonio Liotta

Department of Electronics, Computing and Mathematics, University of Derby, UK

\begin{abstract}
Many distributed services in computer networks rely on a set of active facilities that are selected among a potentially large number of candidates. The active facilities then contribute and cooperate to deliver a specific service to the users of the distributed system. In this scenario graph partitioning or clustering is often adopted to determine the most efficient locations of the facilities. The identification of the optimal set of facility locations is known as the p-median problem in networks, is NP-hard and is typically solved by using heuristic methods. The goal is to select $p$ locations among all candidate network nodes such that some cost function is minimised. A typical example of such a function is the overall communication cost to deliver the service to the users of the distributed system. Locating facilities in near-optimal locations has been extensively studied for different application domains. Most of these studies have investigated sequential algorithms and centralised approaches. However, centralised approaches are practically infeasible in large-scale and dynamic networks, where the problem is inherently distributed or because of the large communication overhead and memory requirements for gathering complete information about the network topology and the users. In this work distributed approaches to the p-median problem are investigated. Two solutions are proposed for addressing the facility locations problem in a fully distributed environment. Two different iterative heuristic approaches are applied to gradually improve a random initial solution and to converge to a final solution with a local minimum of the overall cost. While the first approach adopts a fine granularity by identifying a single change to improve the solution at each iteration, the second approach applies changes to every component of the solution at each iteration. An experimental comparative analysis based on simulations has shown that the approach with a finer granularity is able to deliver a better optimisation of the overall cost with longer convergence time. Both approaches have excellent scalability and provide an effective tool to optimise the facility locations from within the network. No prior knowledge of the system is required, no data needs to be gathered in a centralised server and the same process is used to identify and to deploy the facility locations solution in the network since the process is fully decentralised.
\end{abstract}

\section{Introduction}

Locating facilities in near-optimal locations to deliver some service to users has received a significant interest [1] [2] [3]. A facility is an object or node (such as server or a network device) that provides services such as a distributed memory cache, while users are the network nodes that benefit from the service offered by these facilities. A facility can be either in an open or closed state: an open facility means it can serve connected clients to it, while a closed one is a candidate location where a facility can be opened if required. To provide a more efficient service to the users the topological location of an open facility should be close to many user nodes. The p-median problem, in particular, aims to identify the set of the open facilities (medians) among all the candidate locations such that the overall cost of the solution is minimized according to an objective function [3][4][5]. In many scenarios the cost of the solution is associated to the communication overhead, e.g. the total number of hops in the shortest paths that connect each user node to its closest open facility. This formulation is very similar to the classic partitional clustering problem in data mining, best represented by algorithms such as k-means and k-medoids, where the goal is to find the ideal locations of $k$ centres that minimise the sum of the squared distances from each item to its closest centre.

The main motivation for this work is that previous works on the p-median problem have used a centralised approach based on heuristic methods for finding near-optimal solutions. In that case the required information 
needs to be collected in order to apply a sequential algorithm to find a solution. A centralised approach is infeasible in large-scale networks due to the time and space complexity of the sequential algorithms as well as the large communication cost and latency to aggregate the global information [6, 7, 8]. Therefore, this work investigates distributed algorithms to solve the p-median problem directly within distributed environments.

In this paper, two new approaches for solving the p-median problem in a distributed environment are proposed. Both are designed to be executed without any centralised collection of the data in a single node. They apply an iterative heuristic approach to improve a random initial solution and to converge to a final solution with a local minimum of the cost.

The first approach builds a global view of the system and improves a current solution by replacing a single facility at each iteration. The second approach, is designed on the logic of the k-medoids clustering algorithm. At each iteration, a local view of each cluster is generated and all facilities can be updated to optimise the solution.

Both approaches were implemented in PeerSim, a Java-based network simulator, for investigating the performance in large-scale systems with several tests with different parameters such as the network size, the number of facilities to be located, the total number of candidate facilities and different initial states. The results have shown that the first protocol is more accurate at selecting the locations of the facilities, since it converges to a lower total cost of the solution than the second protocol. However, the second one has shown a better convergence time in optimising the solution.

The rest of the paper is organised as follows. Section 2 introduces the problem, the notation and discusses some related work. Sections 3 and 4 describe the two proposed methods. Section 5 presents the simulations and the comparative experimental results. Finally, Section 6 provides conclusive remarks.

\section{The p-Median Problem Definition, Formulation and Related Work}

The p-median problem intends to find $p$ locations of active (open) facilities among several candidate locations in a way that the total distance (cost) from the user nodes to the open facility nodes is minimised according to an objective function [9] [10] [11]. As most location problems, the p-median problem is classified as NP-hard and solved using heuristic methods [12].

The p-median problem formulation [3] [4] is formally defined as follows. Let us consider a graph $G=(V, E)$, where $V$ is the set of nodes and $E$ the set of links between nodes, the set $F=\left\{f_{1}, f_{2}, \ldots . ., f_{m}\right\}$ of $m$ facilities, the set $U=\left\{u_{1}, u_{2}, \ldots ., u_{n}\right\}$ of $n$ users, where $V=F \cup U$, an integer number $p<m$, which is the target number of open facilities, and a distance function $d: U * F \rightarrow \mathbb{N}$ of the number of hops between users and facilities. A greedy optimisation procedure is applied to build a solution from an initial set of $p$ randomly selected facilities from $F$. The procedure seeks to identify a better neighbour solution to the current one. A neighbour solution is a minimal alteration of the current solution that improves the cost.

A vertex substitution procedure developed by Teitz and Bart [4] is one of the standard algorithms for solving the p-median problem [13] [14]. In vertex substitution, $p$ candidate facilities from $F$ are arbitrarily selected (opened) to start the algorithm. The algorithm reallocates (swap) an open facility with one of the candidate (closed) facilities whenever the swap improves the solution. The algorithm then iterates from the existing solution looking for another pair of facilities to be swapped to improve the solution until the best solution is reached. The algorithm is then terminated with a local optimum solution.

Building up on the vertex substitution algorithm, the fast interchange heuristics algorithm is proposed by Whitaker [15]. It implements a swap once a profitable facilities pair is found. The removed facility is deleted from $F$ and never comes back to the solution [3].

Another study has been done by Hansen and Mladennovic, who used the best improvement strategy in which all possible swaps are evaluated, then the most profitable one is executed. In addition, the way to finding the best facility to open, which is used in the previous method, is evaluated to be less complicated [16].

Based on the above studies, another method to solve the p-median problem was suggested by Resende and Werneck [3]. In their study, all possible swaps were evaluated according to equation 1 and the most profitable pair was chosen for the swap.

$$
\operatorname{profit}\left(f_{i}, f_{r}\right)=\operatorname{gain}\left(f_{i}\right)-\operatorname{loss}\left(f_{r}\right)+\operatorname{extra}\left(f_{i}, f_{r}\right)
$$

As noticed from equation 1 , the first component gain is associated with the candidate facility $f_{i}$ to be inserted in the solution. The gain value is the amount of distance saved by reallocating some user nodes to $f_{i}$, for which $f_{i}$ is closer than their current closest facility. The functions $d_{1}(u)$ and $d_{2}(u)$ are used to indicate the distance from the user node $u$ and, respectively, the closest and second closest open facilities $\phi_{1}(u)$ and $\phi_{2}(u)$. The gain value is computed for all candidates $f_{i}$ according to the equation 2 , so that it results in a vector of size $m-p$. 


$$
\operatorname{gain}\left(f_{i}\right)=\sum_{u \in U} \max \left(0, d_{1}(u)-d\left(u, f_{i}\right)\right)
$$

As a consequence of removing the open facility $f_{r}$ from the solution, users assigned to it must be reassigned to their second closest open facility $\phi_{2}$, which is further away than $f_{r}$ by definition. This leads to an additional cost that is identified by a loss function and is computed according to the equation 3 .

$$
\operatorname{loss}\left(f_{r}\right)=\sum_{u \in U: \phi_{1}(u)=f_{r}}\left[d_{2}(u)-d_{1}(u)\right]
$$

However, some of the user nodes, which were assigned to the removed facility $f_{r}$, are in fact assigned to $f_{i}$ rather than their second closest facility, leading to an additional saving in the total cost. This affects the total cost of the solution and a correction factor called extra profit has to be included for these cases. The extra value is computed as in equation 4 .

$$
\operatorname{extra}\left(f_{i}, f_{r}\right)=\sum_{u \in U:\left[\phi_{1}(u)=f_{r}\right] \wedge\left[d\left(u, f_{i}\right)<d_{2}(u)\right]}\left[d_{2}(u)-\max \left(d\left(u, f_{i}\right), d_{1}(u)\right)\right]
$$

In a distributed environment the information about the network topology, the users and the facilities needs first to be collected in a server in order to compute a solution with a centralised approach. Moreover, once the solution is computed, it will have to be distributed among the nodes in the network for its deployment. This process will then repeat every time there is need to update the solution. In the remainder, two novel distributed protocols, DPM and KM, are presented to solve the p-median problem directly in a distributed environment without prior knowledge about the network topology. No data needs to be gathered in a centralised server and the same process is used to identify and to deploy a p-median solution in the network since the process is intrinsically distributed.

\section{The Distributed p-Median (DPM) Protocol}

As shown in figure 1, the DPM protocol design is based on three main phases to overcome the synchronisation problems of the distributed environment. It is assumed that an initial solution is available, e.g. an initial random solution of $p$ open facilities. The protocol is started with phase 1 by sending BROADCAST messages from all facilities to all nodes in the network. The user nodes are gradually building a view of facilities in the network and determine the closest open facility. Each user node joins its closest open facility via a JoIN message. From the JOIN messages each open facility builds a view of the user nodes assigned to it.

Thereafter, the facility nodes transit to phase 2. In this phase, the open facilities exchange the required information to build a distributed global view about the network and determine the best pair of facilities to swap in the current solution.

In phase 3, the open facilities implement the swap. Both of phases 2 and 3 are repeatedly executed to improve the solution until convergence.

The following subsections describe the details of each phase of the protocol.

\subsection{Phase 1: Initialisation and Information Collection}

In Phase 1 all the candidate (open and closed) facilities disseminate a BROADCAST message to advertise their presence and to facilitate the computation of the shortest paths. This message holds specific information about the facility: ID, status and the distance to reach the facility in number of hops. As described in algorithm 1, from the broadcast messages each user node builds a view about the facilities in the network, which is used to select the closest open facility to join, as further explained in the subsections below.

\subsubsection{Build a user partial view.}

At the start of this phase, the user nodes are kept waiting until receipt of the facility broadcast messages. As soon as a user node receives a new broadcast message, it creates a corresponding local user record by extracting the information from the broadcast message payload, such as the ID of the facility that sends the message, the shortest distance to the facility, the status of the facility and some routing information (e.g., the next hop to reach the facility).

Due to the randomness of the flooding approach for the broadcast messages, the user node may receive a broadcast message from the same facility several times via different paths. If the message comes from a shorter path, the user node updates its local user record, increments the distance field, and forward the message to all its neighbours except the source. 


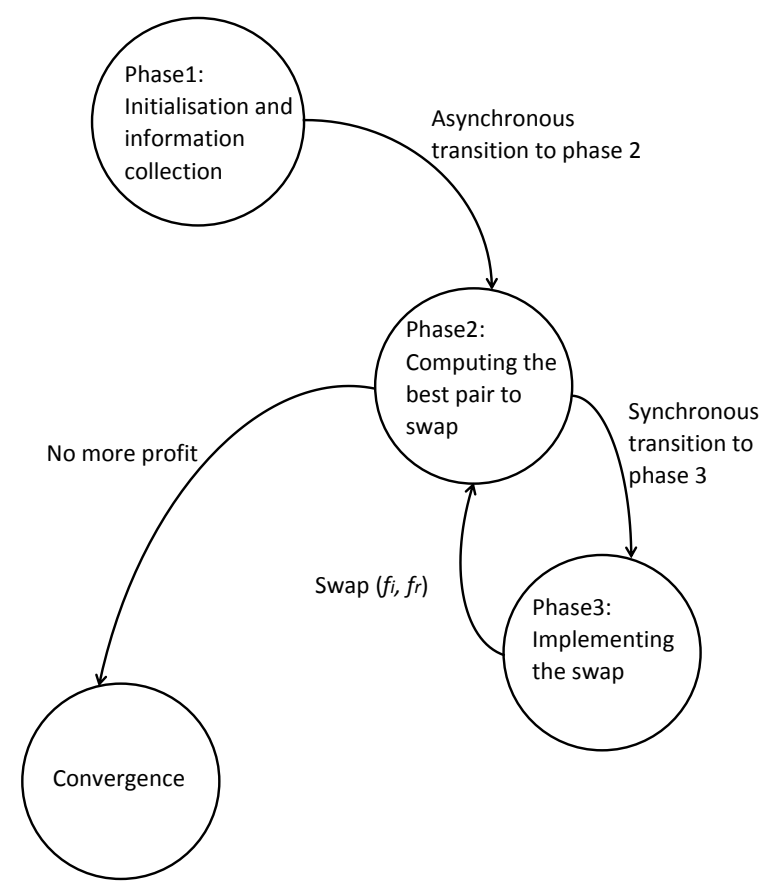

Figure 1: The DPM protocol consists of three main phases. In phase 1 facility information is disseminated over the network and user nodes join their closest open facilities. In phase 2 the open facilities find the best swap of facilities $<f_{i}, f_{r}>$ to minimise the cost. In phase 3 the facilities implement the swap.

\subsubsection{Join the closest open facility.}

While there are no more new messages, the user node computes its loss value, gain and extra vectors. It then sends them to the closest open facility which is determined from its local user record.

When the facility receives the join message from a user node, it updates its local record by adding the user's loss value to the local facility value and the user's gain and extra vector values to their corresponding values in the local facility record, as shown in figure (2). When all join messages are received, the open facilities can transit to phase 2 .

\subsection{Phase 2: Exchange the Necessary Information and Find the Best Pair to Swap}

Each open facility $f_{r}$ is associated to a subset (a cluster) of user nodes, which have identified $f_{r}$ as the closest open facility from which to receive the service.

At the transition to phase 2 , an open facility $f_{r}$ has determined its local value of $\operatorname{loss}\left(f_{r}\right)$ and the two vectors gain $\left(f_{i}\right)$ and $\operatorname{extra}\left(f_{i}, f_{r}\right)$ for the local cluster. In this phase, the open facilities need to exchange and aggregate these local values and vectors to build a global view of the network and of the current p-median solution. Figure 3 shows the collective communication operations (gather and sum), for the aggregation of local information into global information by means of facility exchange messages.

The global loss, gain and extra are initialised with the local values and vectors. When an open facility receives an exchange message containing the remote contribution to the global information, it aggregates the loss of the message into a global loss, adds the gain $\left(f_{i}\right)$ to the global gain vector and collects the extra $\left(f_{i}, f_{r}\right)$ to build a global extra $\left(f_{i}, f_{r}\right)$.

As shown in algorithm 2, when an open facility receives $p-1$ number of exchange messages, it computes the profit values according to the equation 1 for all possible pairs $\left\langle f_{i}, f_{r}\right\rangle$, where $f_{i}$ is a closed facility to be inserted in the solution and $f_{r}$ is an open facility to be removed from the solution. The pair of facilities that provides the greatest profit is chosen for the swap. Based on the shared global view all the open facilities take the same decision by deterministically choosing the same pair of facilities $<f_{i}^{*}, f_{r}^{*}>$ for the swap and thus transiting to phase 3 to implement the swap. 

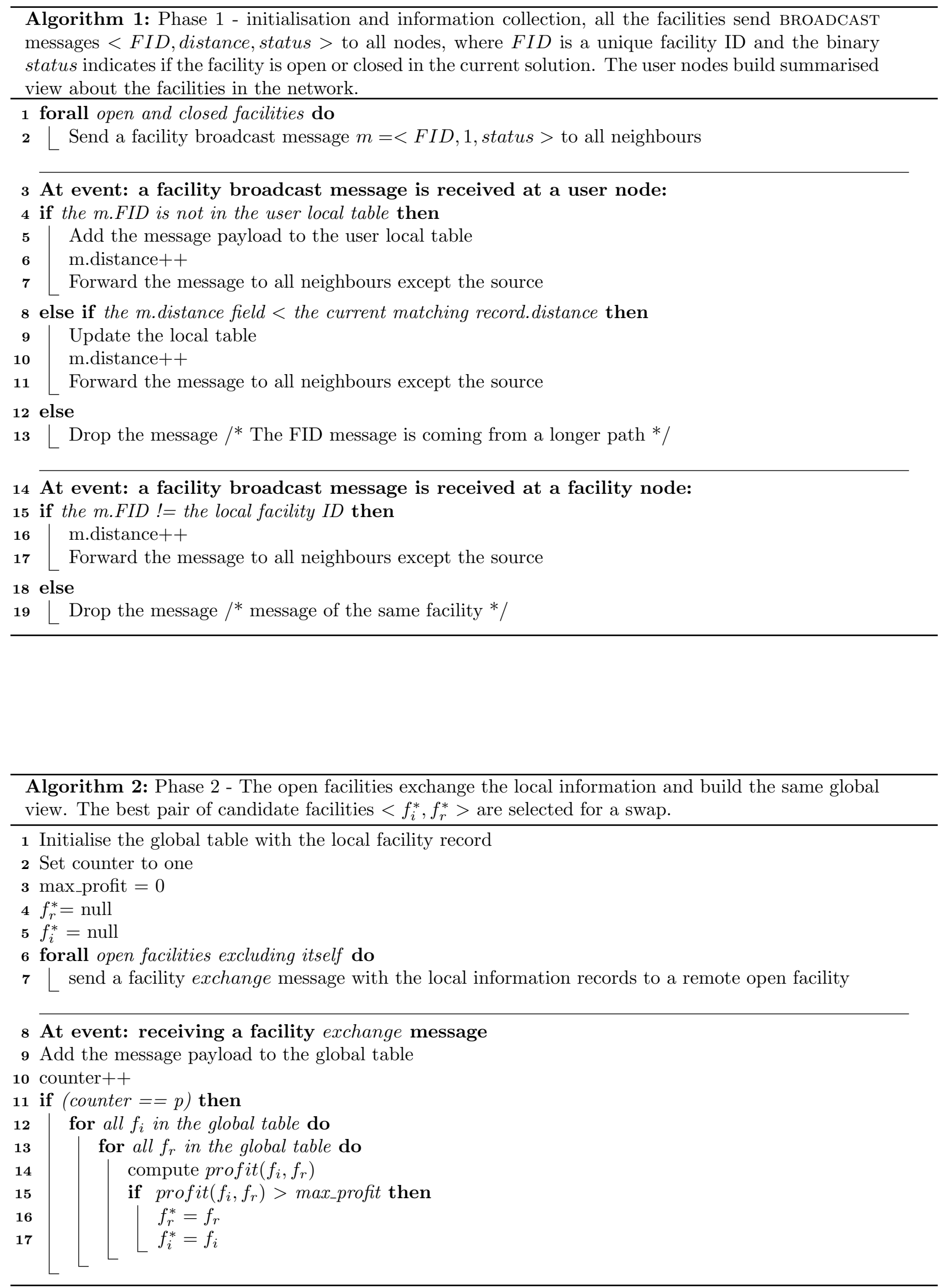


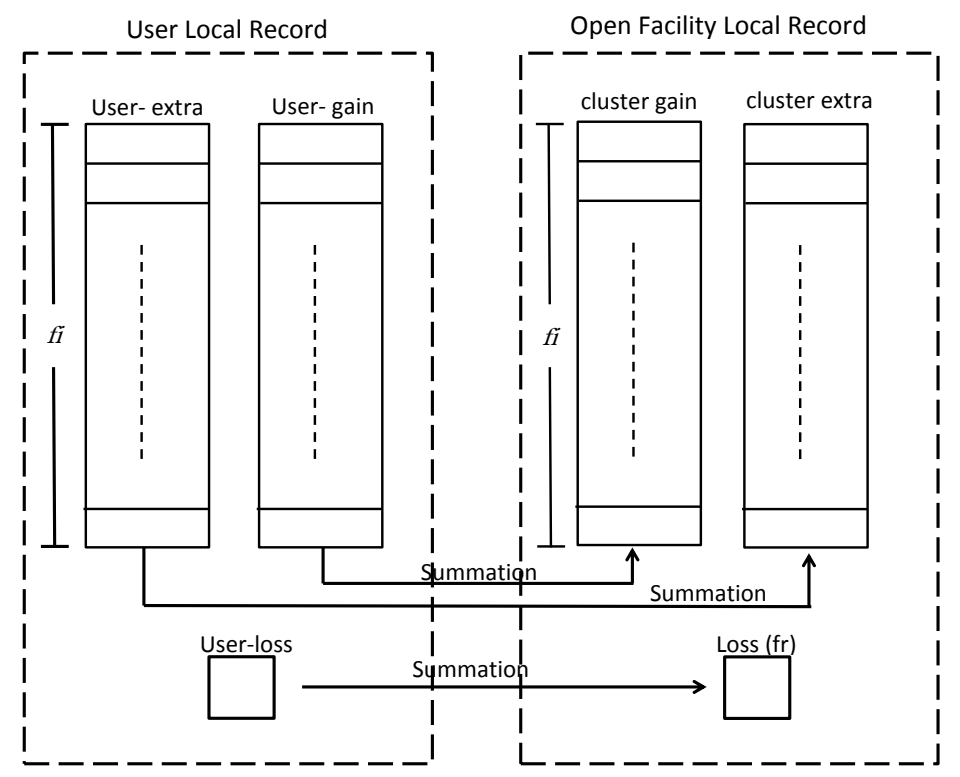

Figure 2: The open facility builds a within-cluster knowledge from the JoIN messages of the user nodes

\subsection{Phase 3: Swap Implementation}

As shown in algorithm 3 , in this phase the candidate $f_{r}^{*}$ sends a CHANGE_STATUS message to $f_{i}^{*}$ asking to change its status to open and to accept join messages from user nodes. The $f_{r}^{*}$ also send a SWAP $<f_{i}^{*}, f_{r}^{*}>$ message to its user nodes (local cluster). If the user node $u$ is closer to the inserted facility $f_{i}^{*}$ than to its second closest open facility $\phi_{2}(u)$, then $u$ sends a join message to $f_{i}^{*}$, otherwise it sends it to $\phi_{2}(u)$.

The current open facilities in the system inform the user nodes in their clusters about the swap by sending an UPDATE_SOLUTION $<f_{i}^{*}, f_{r}^{*}>$ message. If a user node $u$ is closer to $f_{i}^{*}$ than to its current open facility $\phi_{1}(u)$, then $u$ leaves its current cluster and sends a join message to $f_{i}^{*}$.

The solution at this stage is reconfigured. Accordingly, the weight values of the clusters are changed. This may lead to find another pair of facilities to swap in the next iteration to improve the solution further. The process is repeated from phase 2 and the total cost keeps improving until reaching the best (local minimum) configuration for the locations of the candidate facilities, given the initial solution.

\subsection{Convergence}

The convergence status is the final state of the solution, since no more swaps can improve the solution.

\section{The k-Medoids (KM) Protocol}

The second proposed approach is the k-medoids (KM) protocol. This is inspired by the classic Partitioning Around Medoids (PAM), or k-medoids, clustering algorithm [17]. The main idea of the KM protocol is to partition the network around the open facilities, then to iteratively carry out facility swaps with a similar heuristic method used by the k-medoids clustering algorithm. The KM protocol is organised in three main phases, as explained in the subsections below.

\subsection{KM Phase 1: Information Dissemination and Clusters Configuration}

Phase 1 is started by the announcement of the facilities about their locations with broadcast messages similarly to the DPM protocol. Nodes gradually build a summarised view of the available open facilities in the network. They build a local view of facilities, including ID, distance and status. Finally user nodes join the closest open facility forming $p$ clusters. A cluster $C_{k}$ is the set of user nodes associated to the open facility $f_{k}$, such that the distance $d\left(u, f_{k}\right)$ is minimal in the current solution, i.e. $\phi_{1}(u)=f_{k}$.

During this phase, each open facility (medoid) builds a local table of the closed facilities (candidate medoid) and their associated cost before moving to Phase 2, as shown in algorithm 4 . 


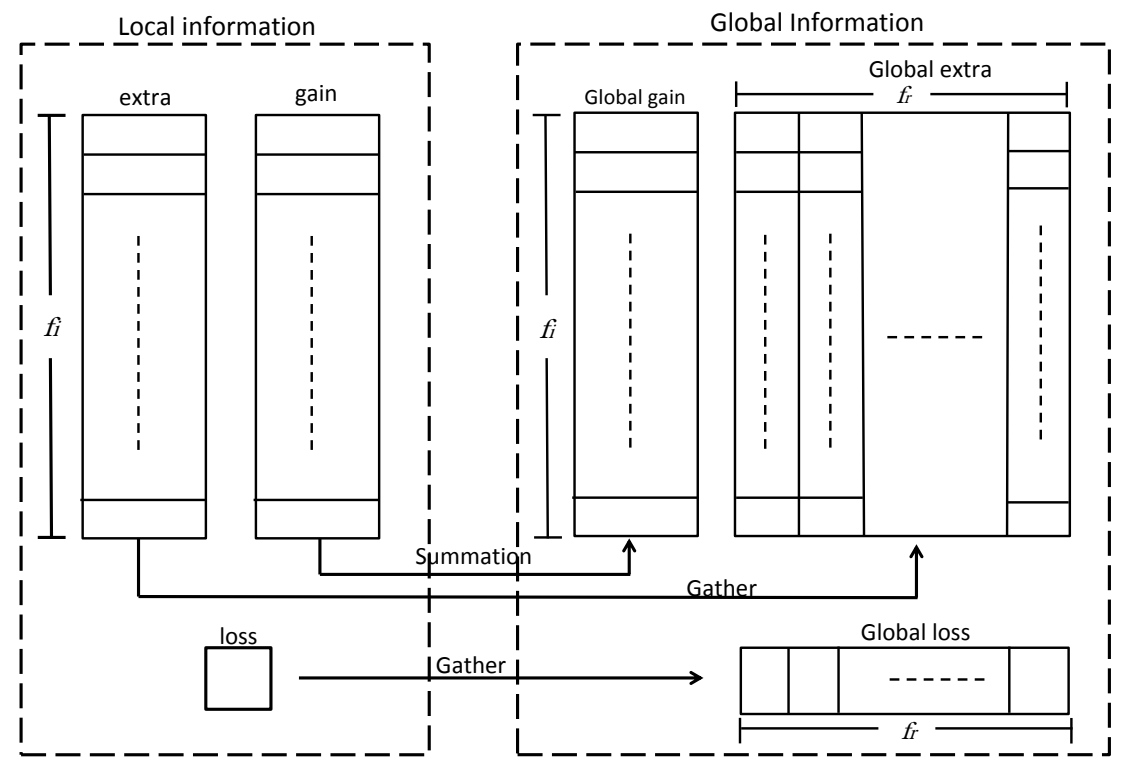

Figure 3: Collective communication operations (gather and sum) among the open facilities for the aggregation of local information to build a global view

\subsection{KM Phase 2: Find the Best $f_{i}$ to Swap}

As shown in algorithm 5 , each medoid $f_{k}$ computes the cost of its cluster $C_{k}$ as the sum of the contributed cost values of the users in the cluster $\left(u \in C_{k}\right)$, as per equation (5). Then it evaluates all the candidate facilities that could be opened within its cluster by computing the cost of these closed facilities as if they would be swapped with the current medoid. The total cost of the solution is given in equation (6). If the cost of one of the candidate facility $f_{i}$ is lower than the cost of the medoid $f_{k}$, then the solution is updated in phase 3 .

$$
\begin{array}{r}
\operatorname{cost}\left(C_{k}\right)=\sum_{u \in C_{k}} d\left(u, f_{k}\right) \\
\operatorname{cost}\left(\left\{C_{k}\right\}\right)=\sum_{0<k \leq p} \operatorname{cost}\left(C_{k}\right)
\end{array}
$$

\subsection{KM Phase 3: Update the medoids of the clusters}

In this phase, as shown in algorithm 6 , all clusters update their medoids independently and in parallel. The update process is implemented as following:

1. The medoid informs the candidate $f_{i}$ with the swap by sending a CHANGE_STATUs message. At the event of receiving the CHANGE_STATUS message; $f_{i}$ changes its status to open and get ready for receiving join messages from both user and closed facility nodes.

2. The medoid informs the users and the closed facilities in its cluster of the swap by sending them a SwAP $<f_{i}, f_{r}>$ message. The user and the closed facility nodes update their local table by changing the status of $f_{i}$ to open and the status of $f_{r}$ to closed, determine a better, if any, closest open facility to join.

3. The medoid informs all other open facilities in the system of the swap by sending CLUSTER_UPDATE $<f_{i}$, $f_{r}>$ message to the medoids of all other clusters. At the event of receiving CLUSTER_UPDATE $<f_{i}, f_{r}>$ message, the medoid informs the user and closed facilities nodes in its clusters about the swap.

\subsection{Convergence State}

Phases 2 and 3 are repeatedly executed and the clusters medoids are updated continuously until no more update messages are received: in this case the facilities make a transition into the convergence state and the algorithm is terminated in a solution with a locally optimal cost. 


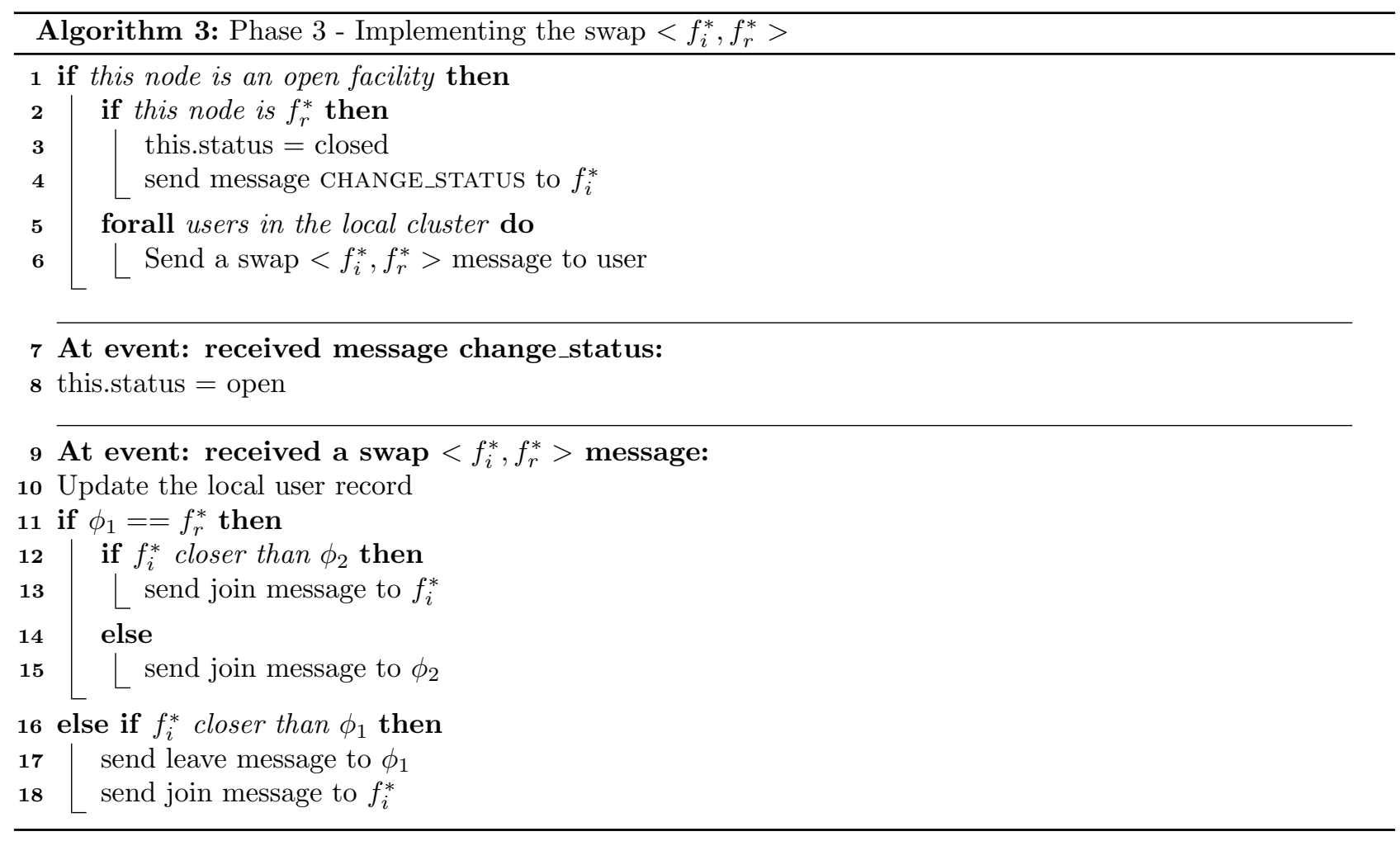

\section{$5 \quad$ Simulation and Experimental Results}

A Java-based discrete-event P2P simulation tool called PeerSim [18] is used to simulate the proposed protocols. PeerSim allows simulating large networks with different configurations. In addition to its ability to observe the internal state of nodes in the simulated topology, it allows to keep track of the messages between the facilities and the user nodes. This aids in the performance analysis of the protocols.

The DPM and KM protocols are extensively tested over a range of the network size (up to $500 K$ nodes) of artificial and real network topologies.

Figure 4(a) shows the run of both protocols until convergence: the mean and the standard deviation over 10 trials with different randomly chosen initial solutions are shown. The results demonstrate a reduction on the overall cost of the solution at each iteration (swap) until convergence for both protocols. However, KM protocol shows a higher cost of the solution during all cycles. This confirms that the DPM protocol based on global knowledge is more effective in improving the solution cost than the KM protocol, which improves the location of the facilities based on local cluster information.

As shown in figure 4(b), the KM protocol converges faster than the DPM protocol. However, the DPM protocol is found to be capable to identify better final locations for the facilities.

For testing the DPM and KM on different graphs, many trials were carried out on different network sizes (figure 5(a)): the mean cost and the standard deviation of these trials showed for all graph sizes that the DPM protocol provides a lower cost than the KM protocol.

Both protocols are also tested on a varying number $(m)$ of the available candidate locations for the facilities on the same topology. As shown in figure 5(b), DPM takes better advantage than KM of the larger search space for the solution of the p-median problem.

Since both the DPM and KM protocols depend on network messages for addressing the p-median problem, the communication overhead in terms of the number of messages is analysed for the different types of messages. As shown in figure 6, the most significant number of messages among the nodes is the facility BROADCAST messages. These messages flood the network to propagate information from all facilities to all user nodes.

Practically, each facility is meant to serve nodes in its cluster, typically a local part of the topology. Further investigation has shown that it is unnecessary to forward the broadcast messages to the whole network since it causes a heavy load on the network and an unnecessary delay. Instead, the outreach of the broadcast messages can be restricted to a maximum number of hops (limited time-to-live). This restriction is not affecting the performance of the protocols, while it is significantly reducing the communication cost, as shown in figure 6 . 

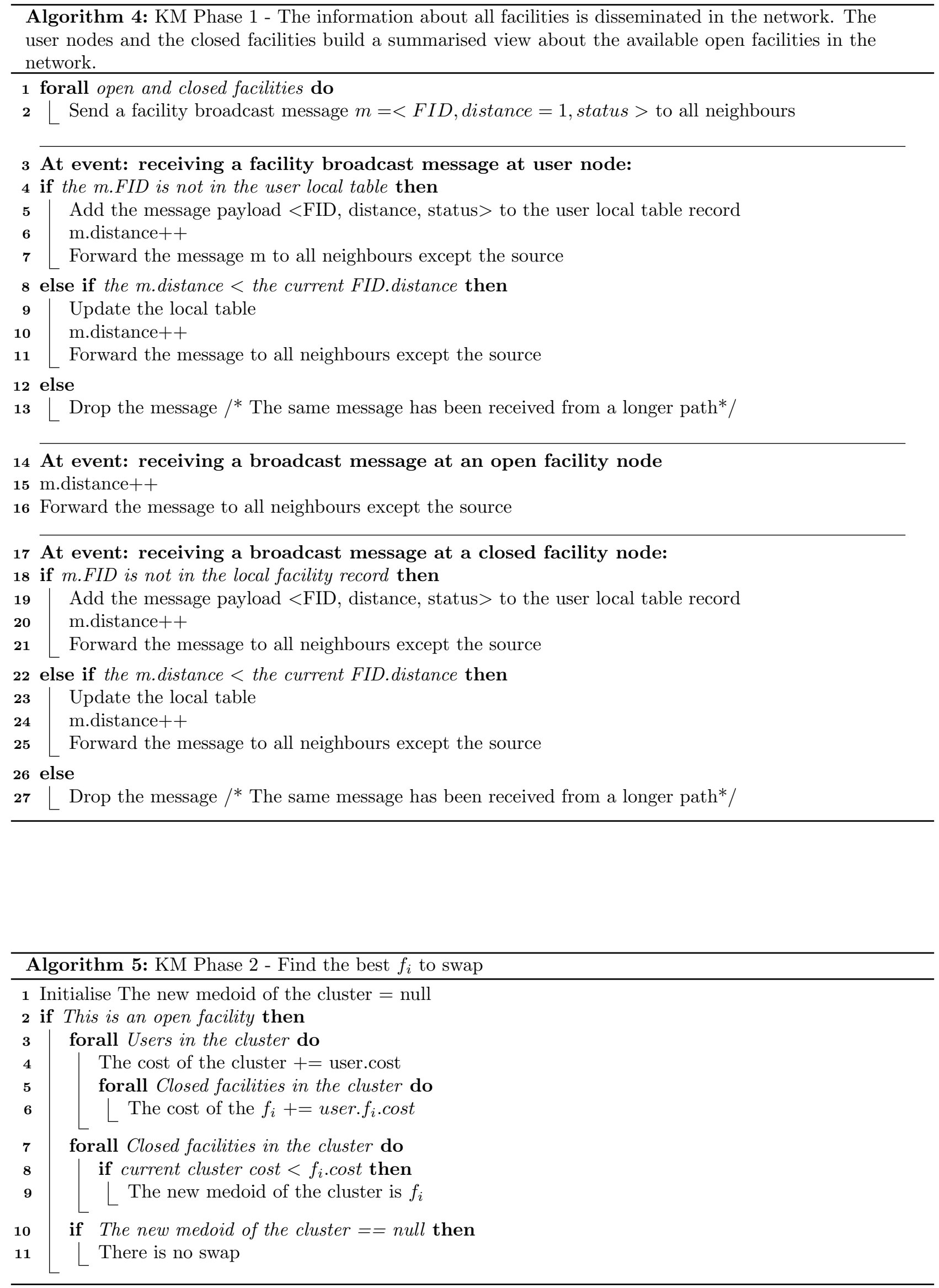


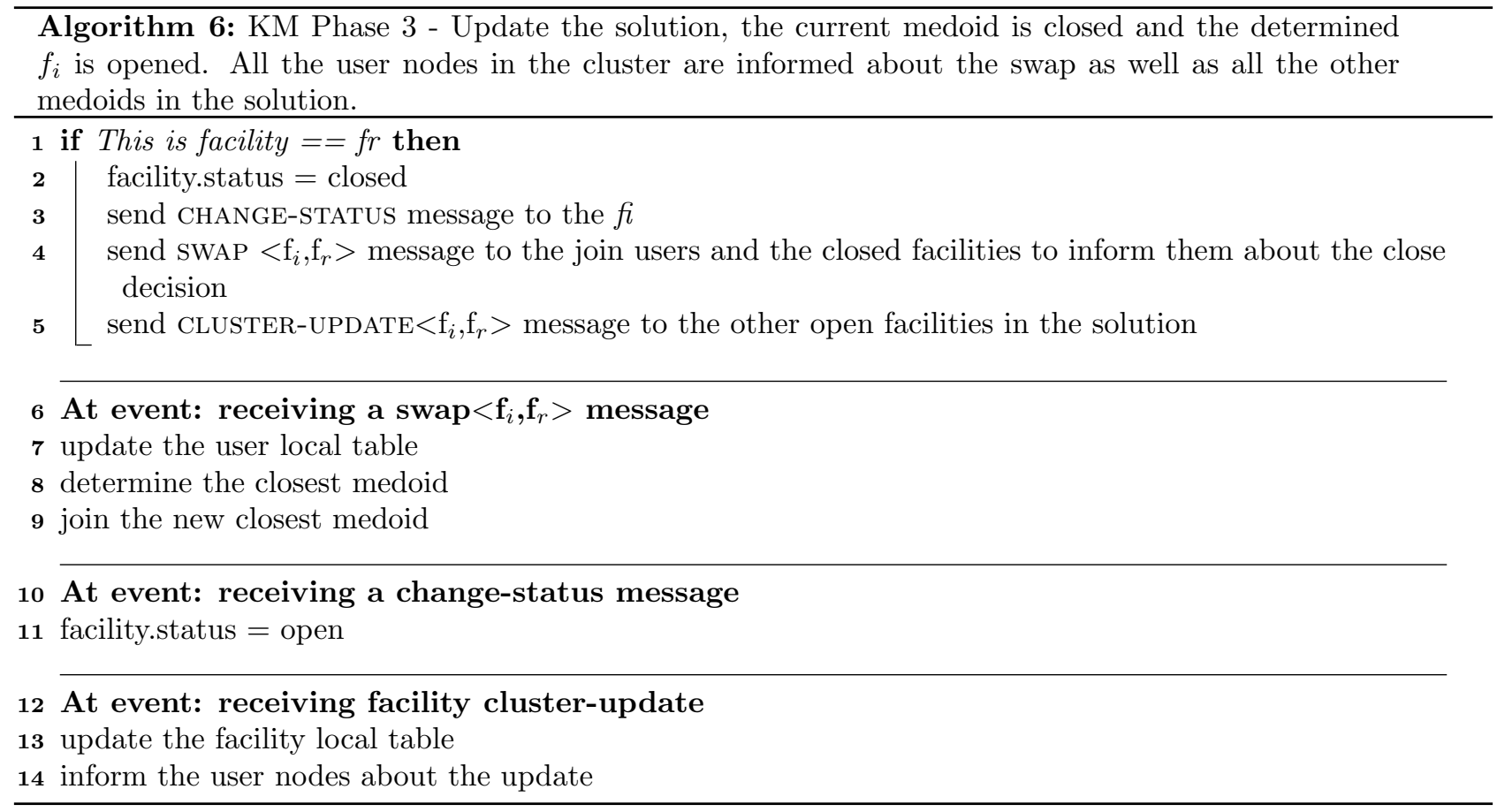

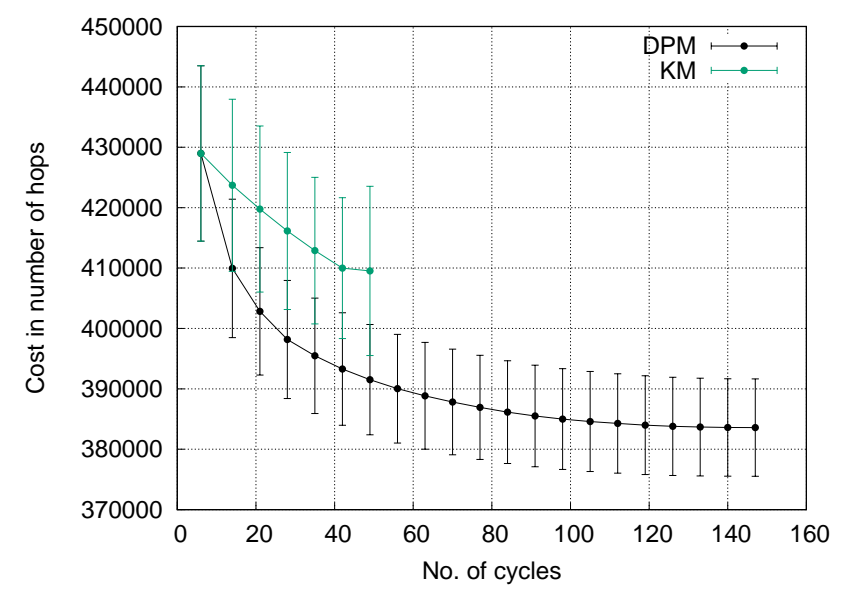

(a)

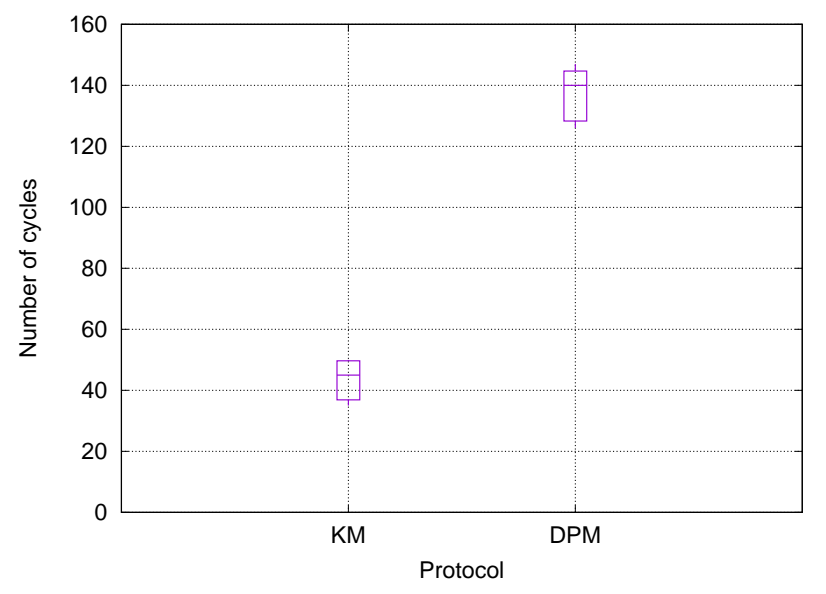

(b)

Figure 4: Convergence analysis: mean and standard deviation over 10 trials $(\mathrm{N}=100 \mathrm{~K}, \mathrm{~m}=100, \mathrm{p}=25)$

\section{Conclusions}

This paper has presented two novel distributed approaches for the p-median problem in networks. The proposed methods are executed without a prior knowledge of the network topology. Network information, such as the topology and the set of candidate facilities and users, do not need to be gathered for a centralised execution of a sequential algorithm. The computation in the proposed protocols is intrinsically distributed in the network nodes.

A comparative analysis over many simulations has revealed that addressing the location problem for facilities based on a global view of the network (as in DPM) leads to more accurate optimisation of the cost than clustering the network and optimising each facility location based on the local view of each cluster separately (as in KM). However, the former solution takes more cycles to converge.

The simulations have also shown that when more candidate facilities are available, both protocols can find a better solution in terms of cost. Also in this case DPM has confirmed to have better performance in taking advantage of more locations. However, when more locations are available both protocols require additional time to reach convergence. 


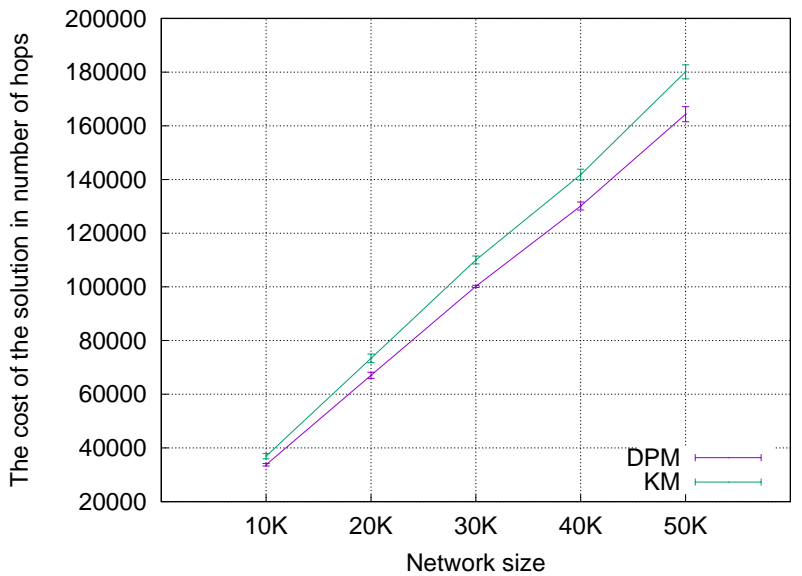

(a) Different network size

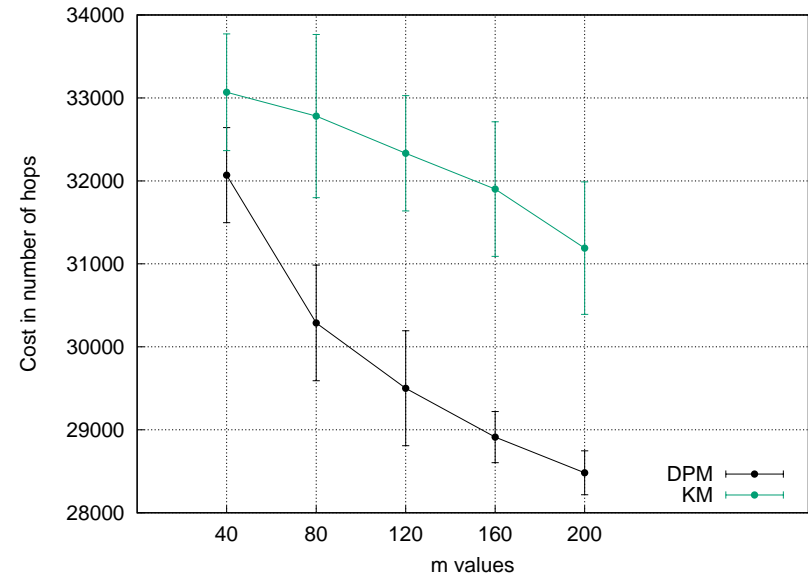

(b) Different number of candidate facilities $m(p=25)$

Figure 5: Cost (mean and standard deviation) of the solution at convergence

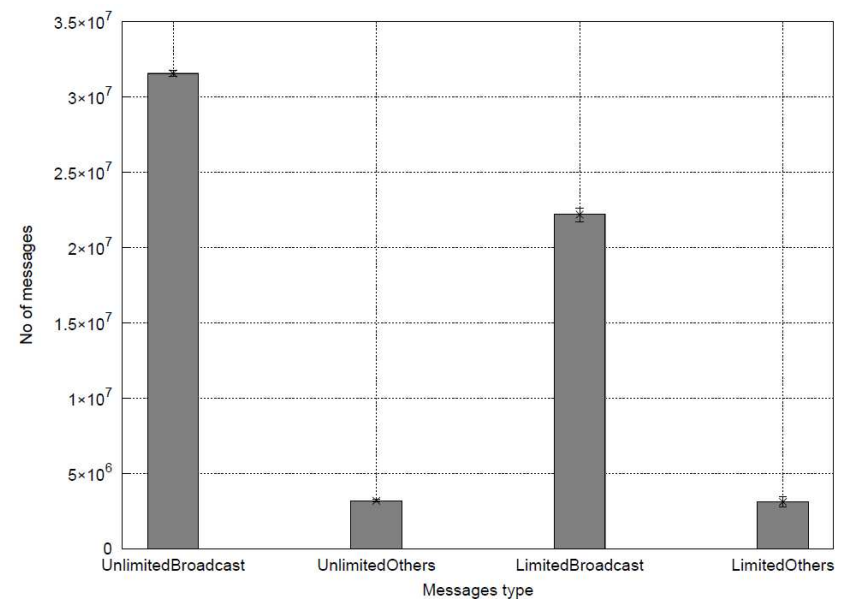

(a) DPM protocol

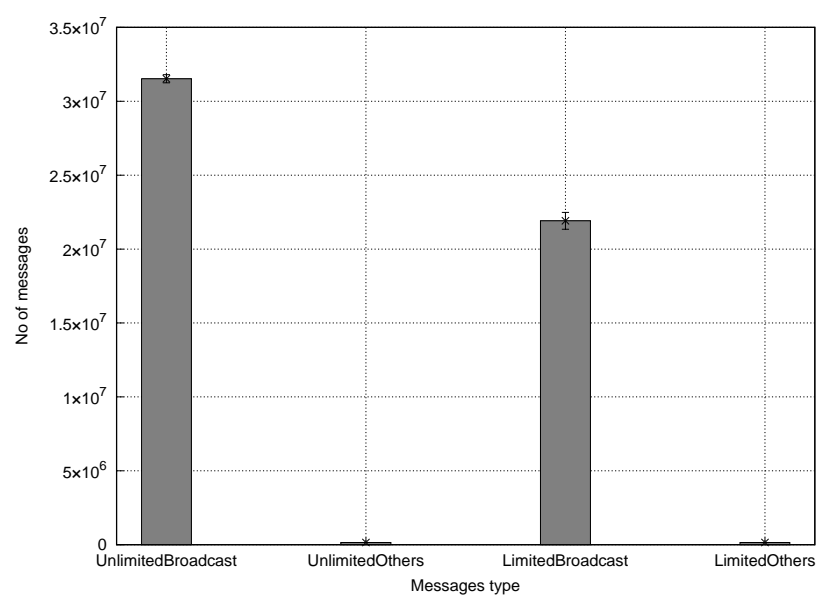

(b) KM protocol

Figure 6: Comparison of communication overhead for broadcast and other types of messages, and for unlimited and limited (maximum time-to-live) message propagation $(\mathrm{N}=50 \mathrm{~K}, \mathrm{~m}=100, \mathrm{p}=25)$

\section{References}

[1] J. Alcaraz, M. Landete, and J. F. Monge. "Design and analysis of hybrid metaheuristics for the reliability p-median problem". In: European Journal of Operational Research 222.1 (2012), pp. 54-64.

[2] M. T. Melo, S. Nickel, and F. Saldanha-Da-Gama. "Facility location and supply chain management-A review". In: European journal of operational research 196.2 (2009), pp. 401-412.

[3] M. G. Resende and R. F. Werneck. "A fast swap-based local search procedure for location problems". In: Annals of Operations Research 150.1 (2007), pp. 205-230.

[4] M. B. Teitz and P. Bart. "Heuristic methods for estimating the generalized vertex median of a weighted graph". In: Operations research 16.5 (1968), pp. 955-961.

[5] T. S. Hale and C. R. Moberg. "Location science research: a review". In: Annals of operations research 123.1-4 (2003), pp. 21-35.

[6] H. Mashayekhi, J. Habibi, T. Khalafbeigi, S. Voulgaris, and M. Van Steen. "GDCluster: a general decentralized clustering algorithm". In: IEEE transactions on knowledge and data engineering 27.7 (2015), pp. 1892-1905.

[7] G. Di Fatta, F. Blasa, S. Cafiero, and G. Fortino. "Epidemic K-Means Clustering". In: 2011 IEEE 11th International Conference on Data Mining Workshops. 2011, pp. 151-158.

[8] G. Di Fatta, F. Blasa, S. Cafiero, and G. Fortino. "Fault tolerant decentralised K-Means clustering for asynchronous large-scale networks". In: Journal of Parallel and Distributed Computing 73.3 (2013), pp. 317329. 
[9] O. Alp, E. Erkut, and Z. Drezner. "An efficient genetic algorithm for the p-median problem". In: Annals of Operations research 122.1-4 (2003), pp. 21-42.

[10] M. Labbé, D. Ponce, and J. Puerto. "A comparative study of formulations and solution methods for the discrete ordered p-median problem". In: Computers 6 Operations Research 78 (2017), pp. 230-242.

[11] M. Karatas, N. Razi, and H. Tozan. "A Comparison of p-median and Maximal Coverage Location Models with Q-coverage Requirement". In: Procedia Engineering 149 (2016), pp. 169-176.

[12] V. Marianov and D. Serra. "Median problems in networks". In: Foundations of location analysis. Springer, 2011, pp. 39-59.

[13] A. I. Mahmutogullari and B. Y. Kara. "Hub location under competition". In: European Journal of Operational Research 250.1 (2016), pp. 214-225.

[14] M. J. Hodgson, F. Shmulevitz, and M. Körkel. "Aggregation error effects on the discrete-space p-median model: The case of Edmonton, Canada". In: Canadian Geographer/Le Géographe canadien 41.4 (1997), pp. $415-428$.

[15] R. Whitaker. "A fast algorithm for the greedy interchange for large-scale clustering and median location problems". In: INFOR: Information Systems and Operational Research 21.2 (1983), pp. 95-108.

[16] N. Mladenović, J. Brimberg, P. Hansen, and J. A. Moreno-Pérez. "The p-median problem: A survey of metaheuristic approaches". In: European Journal of Operational Research 179.3 (2007), pp. 927-939.

[17] L. Kaufman and P. Rousseeuw. "Clustering by means of Medoids". In: Statistical Data Analysis Based on the $L_{1}$ Norm and Related Methods. 1987, pp. 405-416.

[18] A. Montresor and M. Jelasity. "PeerSim: A scalable P2P simulator". In: Peer-to-Peer Computing, 2009. P2P'09. IEEE Ninth International Conference on. IEEE. 2009, pp. 99-100. 